\title{
红外成像系统中自动对焦功能优化方法研究
}

杨瑞宇，吕浩，龚晓霞，吴诚，李学宽，苏兰，范明国，尹敏杰，杜润来 (昆明物理研究所, 云南 昆明 650223)

\begin{abstract}
摘要: 为了在红外成像系统中实现高效的自动对焦功能, 深入分析和讨论了红外成像系统中自动对焦 功能的优化方法及特点。结合具体的工程化应用, 提出了一种对图像清晰度评价函数局部随机起优噪 声的估计方法, 该方法可以提高自动对焦功能的可靠性, 通过将图像清晰度评价函数的灵敏度作为反 馈量引入爬坡过程，优化了爬坡算法的收敛速度。其优化方法在工程应用中的实施效果，验证了设计 的正确性，优化方法的有效性。
\end{abstract}

关键词: 自动对焦; 红外成像; 图像清晰度评价函数; 爬坡算法; 自动对焦窗口

中图分类号：TP751 文献标识码：A 文章编号：1001-8891(2020)10-0940-07

\section{Optimizing Method of Autofocusing Function in Infrared Imaging System}

\author{
YANG Ruiyu, LYU Hao, GONG Xiaoxia, WU Cheng, LI Xuekuan, SU Lan, FAN Mingguo,
} YIN Minjie, DU Runlai

(Kunming Institute of Physics, Kunming 650223, China)

\begin{abstract}
In order to realize high efficiency auto focusing function in infrared imaging system, the characteristics of infrared imaging systems are analyzed. The optimization methods of auto-focusing functions in infrared imaging systems are discussed and summarized. Combined with practical engineering applications, a method for estimating the local random fluctuation noise of the image sharpness evaluation function is proposed. The method improves the reliability of automatic focusing processes. By introducing the sensitivity of image sharpness evaluation function as feedback into the climbing process, the convergence speed of the climbing algorithm can be optimized. The optimization methods were applied to engineering applications, and its implementation verified the correctness of the design and the effectiveness of the optimization methods.
\end{abstract}

Key words: auto-focusing, infrared imaging system, image sharpness evaluation function, mountain-climb searching algorithm, focus window

\section{0 引言}

红外成像技术作为光电成像技术的重要分支学 科, 其工程化的应用极大地拓展了人们的 “视野”, 成为了人类征服未知 “漆黑”世界的一件 “利器”。 但受制于价格因素, 目前阶段主要的应用, 仍以军事 运用背景为主, 通常用于大型武器装备的侦查、稳瞄, 或夜间导航，辅助驾驶等。在考虑到不增加红外系统 复杂性前提下, 基于数字图像信息处理的对焦深度自 动对焦方法, 成为了为一种高性价比、务实可行的自 动对焦功能实现方案 ${ }^{[1]}$ 。

采用基于数字图像信息处理的对焦深度方法, 实 现自动对焦功能, 主要包含 3 个关键技术, 一是清晰
度评价函数, 二是对焦窗口的选择, 三是搜索算法。 而自动对焦功能的优化实现过程, 具体方法通常围绕 着基于数字图像信息处理的对焦深度方法的 3 个关键 技术而展开。

图像清晰度评价函数可分为多种, 分别是灰度函 数、频域函数、信息熵函数、统计学函数和基于边缘 轮廓线提取函数等 ${ }^{[2-5]}$ 。清晰度评价函数的选取应满足 如下设计约束性要求：(1)有效性：运算得到的对焦位 置要和实际的焦点位置表现出重合，而不是一个无关 的位置; (2)单峰性：该函数在调焦的有效行程中有且 仅有一个极值位置, 对应着自动调焦的最优位置（或 算法自身能剔除双峰或多峰带来的歧义); (3)无偏性: 该函数对不同目标背景有着类似的响应率分布（针对 
调焦行程), 对不同目标背景计算得到的曲线要与场 景的实际变化相吻合。选取的清晰度评价函数, 应 满足一定的性能要求: (1)高灵敏度: 该函数要在峰 值附近随焦距的变化表现出明显的数值变化, 以方 便确定峰值的位置; (2)高信噪比: 该函数计算方法 对图像噪声和目标背景的微小扰动的影响有一定抗 干扰能力 ${ }^{[5-6]}$ 。通过选择合理的对焦窗口可以降低评 价函数运算量, 剔除不同景深下对同一背景目标带来 的影响 ${ }^{[7]}$ 。对焦窗口的选择方案有: 中央选择法、多 区域选择法、黄金分割点位置法和神经算法等 ${ }^{[8]}$ 。

搜索算法决定于控制系统, 能否在自动对焦过程 中, 根据当前图像清晰度评价函数的值, 控制焦距变 化以快速收敛至最佳对焦位置。常用的对焦搜索算法 有盲人爬坡法、穷举搜索法、Fibonacci 搜索法、函数 逼近法和黄金分割法等。具体实现应重点考虑如下 3 个要素: (1)合理初值的选取; (2)收敛的有效性; (3)高 的收敛速度 ${ }^{[9-10]}$ 。

大型武器装备中的红外成像系统中, 视距通常是 其系统功能追求的首要指标, 但探测器往往匹配于复 杂而笨重的光学成像机构, 在此类红外成像系统中实 现自动对焦功能, 存在一定实施的困难。通常的具体 表现有: 视场变倍比大 (调焦行程较长)、调焦结构 载荷重 (执行机构运动迟缓)、齐焦性能随温度漂移 较大、和红外图像性噪比低等一系列特点, 最终将导 致自动对焦功能实时性能下降。本文针对上述红外成 像系统的特点, 通过研究自动对焦功能的关键技术, 提出了一种基于数字图像信息处理的自动对焦深度 优化方法, 在某一型号无人机红外载荷自动对焦功能 的实现过程中, 通过成功率、精度和速度 3 个关键性 能参数的测试和评价, 验证了其优化方法的有效性。

\section{1 自动对焦关键技术的优化}

\section{1 图像清晰度评价函数优化}

清晰度评价函数提取方法有多种, 其中基于边缘 特性提取的方法有: 常规的离散差分 (differentiation) 算子、基于微分的 Prewitt 算子、常规的梯度算子 （gradient）、基于梯度的 Roberts 算子、Sigma 算子和 Sobel 算子等。为了比较不同方法提取清晰度评价函 数的效果, 本文通过实验方法采集计算了上述算子计 算得到的图像清晰度评价函数的实验数据, 和与之对 应的不同调焦位置的拟合曲线图，如图 1 所示。

在图 1 中, 通过 NI（National Instruments）公司 的机器视觉软件, 采样计算了某型号热像仪的上述 6 种不同算子得到的图像清晰度评价函数拟合曲线图, 从图中我们不难发现其中的 Sobel 算子提取图像清晰
度评价函数的拟合曲线较其他 5 条拟合曲线, 相对平 滑 (局部噪声波动较小, 图像清晰度评价函数拟合曲 线的归一化均方根值较小), 同时峰值绝对值较大 (图 像清晰度评价函数多项式拟合趋势图的峰值梯度), 单 调性较好 (图像清晰度评价函数多项式拟合趋势图的 平均梯度较大), 见表 1 。在实际情况下, 由于噪声的 影响, 对局部最大值与最小值的确定就存在较大的误 差, 从而导致图像细节模糊、不清晰。从图 1 的对比 分析中我们可以得出结论: 针对于实验中涉及的红外光 电系统, 上述的 6 种不同算子, Sobel 算子更适合作为 此红外成像系统中图像清晰度评价函数的提取方法。

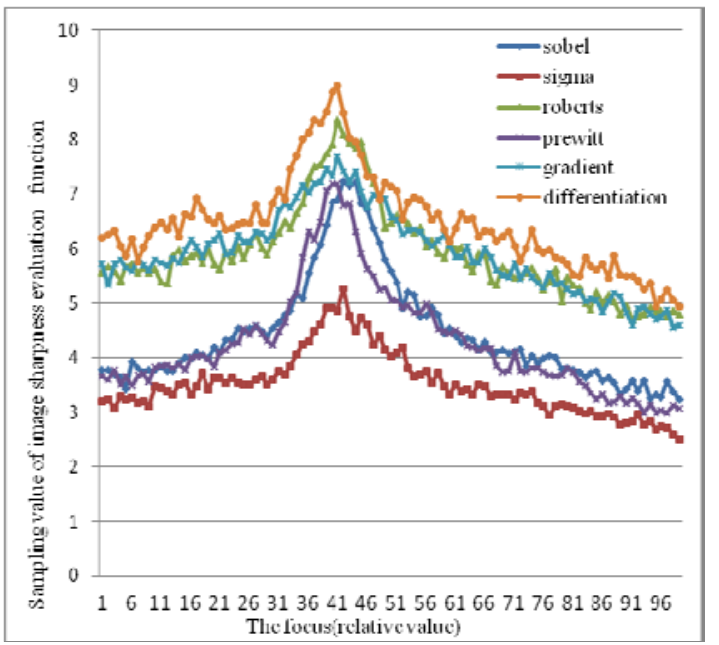

图 1 多种方法提取热像清晰度评价函数拟合曲线的比较图

Fig.1 Comparison chart of fitting curves of thermal image sharpness evaluation function extracted by various methods

在红外数字图像视频流处理过程中, 合理选择提 取图像清晰度的节点, 是自动对焦功能优化方法的一 个技术关键点。图 2 为某型号机载热像仪所拍摄得到 的实物和经过数字滤波处理前后的图像及相应的图 像清晰度评价函数 (随焦距) 曲线的对比图, 其中图 2(a)为某型号机载热像仪数字滤波处理前的图像场景 图, 该图有较多盲点, 看起来较为模糊、清晰度较差; 图 2(b)为某型号机载热像仪数字滤波处理后的图像场 景图, 在图 2(a)的基础上, 我们引入了数字滤波处理, 从处理前后的两幅对比图中可以很直观地看到, 经过 处理后的图像, 剔除了原有图像中的较多盲点, 整体 提高了图像的质量和清晰度。

图 2(c)和图 2(d)为与 2(a)和 2(b)相对应的图像清 晰度函数值拟合曲线, 从图 2(a)中可以明显看出, 在 该图中存在较多的高频椒盐噪声。结合该图我们来讨 论椒盐噪声对图像清晰度函数（此处, 定义为 $D$ ) 的 影响。对图 2(c)和图 2(d)进行认真观察和仔细比较后, 可以发现 2 幅图像的清晰度函数值拟合曲线无论在 形状上或者是整体趋势上变化均不大, 主要的差异表 
现在于：图 2(d)较图 2(c)增加了一个相对固定的线性 偏置分量。其结果是: 由于图 2(b)的椒盐噪声产生的 线性偏置分量被滤除 (或得以抑制), 导致与之对应 的曲线有较大相对变化率。
就上述情况，考虑到图像清晰度函数的灵敏度的 影响, 图 2(b)较图 2(a)更适合于自动对焦功能的实现。 由此可见，在红外成像系统中为保证自动对焦功能的 实现和性能的提升，往往更加期望一个 “干净”（无

表 1 多种图像清晰度评价函数计算方法的对比表

Table 1 Comparison of several methods for calculating image sharpness evaluation function

\begin{tabular}{lccc}
\hline $\begin{array}{l}\text { Calculation method } \\
\text { of image sharpness } \\
\text { evaluation function }\end{array}$ & $\begin{array}{l}\text { The peak gradient of trend } \\
\text { graph fitted by polynomial for } \\
\text { image sharpness evaluation } \\
\text { function(absolute value) }\end{array}$ & $\begin{array}{l}\text { The average gradient of trend } \\
\text { graph fitted by polynomial for } \\
\text { image sharpness evaluation } \\
\text { function(absolute value) }\end{array}$ & $\begin{array}{l}\text { The noise estimation of fitting } \\
\text { curve for image definition } \\
\text { evaluation function(normalized } \\
\text { root-mean-square value) }\end{array}$ \\
\hline Sobel & 0.78 & 0.132 & 0.55 \\
Sigma & 0.73 & 0.132 & 0.88 \\
Roberts & 0.76 & 0.131 & 0.76 \\
Prewitt & 0.75 & 0.122 & 0.67 \\
Gradient & 0.77 & 0.109 & 0.92 \\
Differentiation & 0.74 & 0.125 & 1.03 \\
\hline
\end{tabular}

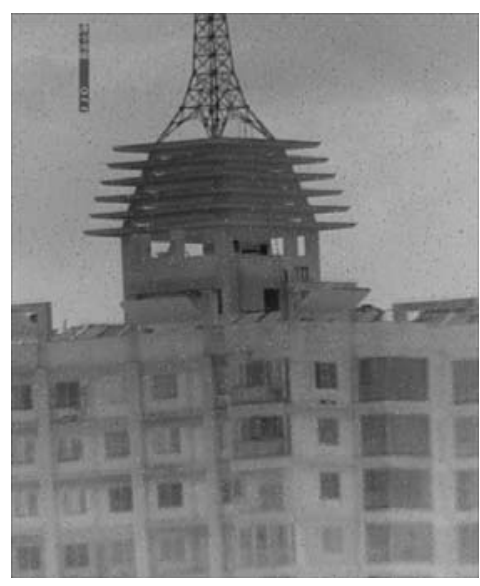

(a) 数字滤波前的图像

(a) Image before digital filtering of thermal image

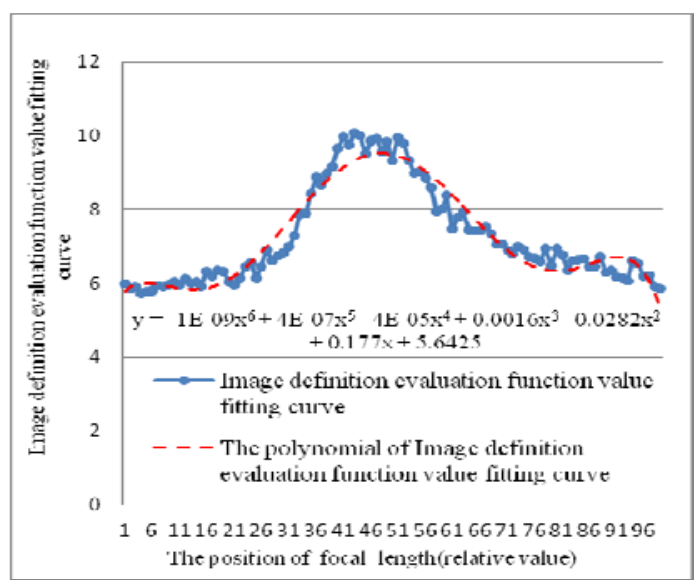

(c) 滤波前的图像清晰度评价函数曲线

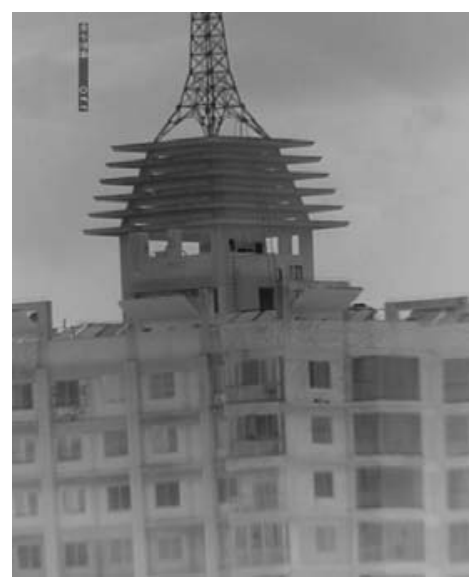

(b) 数字滤波后的图像

(b) Digital filtered image of thermal image

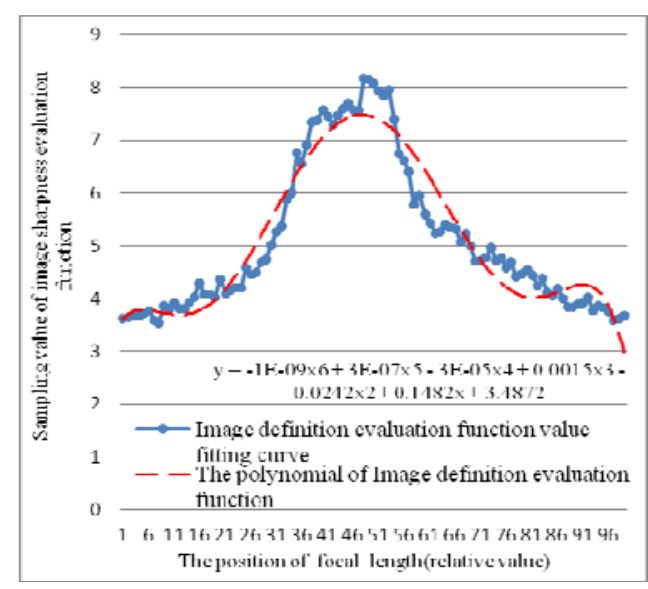

(d) 滤波后的图像清晰度评价函数曲线

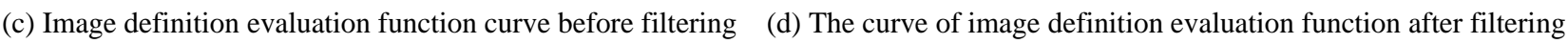
图 2 热像数字滤波前后的图像及相应的图像清晰度评价函数的曲线

Fig.2 The thermal image before and after digital filtering vs. the corresponding curve of image definition evaluation function 
噪声干扰）的场景。红外成像数字图像处理中, 除了 特有的非均匀校正算法和盲元替换算法, 为了解决目 标场景 (或采样电路输入) 与输出观瞄显示系统的动 态范围不匹配的问题, 往往还会引入一些非线性动态 压缩和细节增强算法, 此类非线性的处理, 无疑将增 加清晰度评价函数的选取违背自动对焦设计约束的 风险 (有效、单峰、无偏性)。兼顾考虑到其要求的 性能参数: 高性噪比和灵敏度, 本文选择了经过非均 匀校正, 滤除图像本征的高频噪声, 进行盲元替换后, 实时直方图均衡前的视频流, 作为图像清晰度评价函 数的提取节点。本文所涉及红外成像视频的数据流 图, 如图 3 所示。

\section{2 自适应的对焦窗口调整}

动态自适应地选择对焦窗口, 更有利于得到单峰 的图像清晰度评价函数。假设 $F_{N}(N$ 取自然数, 代表 第 $N$ 次调焦的计数) 为调焦过程中焦距相对位置序列, 与之相对应的图像清晰度评价函数值, 也记为 $D_{N}$ 。本 文涉及的具体工程实践中, 通过细分子窗口, 并根据 图像清晰度评价函数调焦步长改变的变化量 $\Delta D$ (其 中, $\Delta D=D_{N}-D_{N-1}$ ), 可以选择 $\Delta D$ 较大的子窗口, 作 为清晰度评价函数计算中采样计算数据窗口, 以实现 本部分的设计方法的优化。如图 4, 如将中心窗口进
一步细分 16 个子窗口, 其中图 4(c)和图 4(d), 是图 4(a)和图 4(b)相对应图像, 进行拉普拉斯滤波后的图 像。在大视场情况下, 不难发现子窗口 $\mathrm{W}_{00}, \mathrm{~W}_{01}$, $\mathrm{W}_{10}, \mathrm{~W}_{30}$ 和 $\mathrm{W}_{31}$ 对于图像清晰度评价函数的总体变 化量的贡献量是有限的。此种情况下, 选择计算的 子窗口可以不包括上述子窗口; 而在小视场情况 下, 情况则相反。所以, 在自动对焦前或过程中动 态、自适应地选取有效的对焦窗口, 也是一种优化 自动对焦功能的有效方法。

\section{3 噪声的估计和爬坡算法的优化}

爬坡算法的优化, 一直是红外成像系统中实现自 动对焦技术的难点和研究热点。近年来, 林忠、王剑 华和何炳阳等人提出了 “两次下降爬坡算法” ${ }^{[2]}$,

“变调焦步进的爬坡算法” ${ }^{[10]}$ 和 “自适应的自动对 焦算法” ${ }^{[1]}$, 其要解决的问题是如何使爬坡算法能 克服局部峰值, 但大多数算法通常是增加了爬坡算 法的㝋余度（可能降低算法收玫速度）或加强了算法 实现约束的条件 ${ }^{[11]}$, 在具体工程应用的情况中, 也 存在一定的实现难度。本文在某型号无人机红外观 瞄热像系统的工程化实现中, 设计如下自动对焦技 术的优化方法, 以实现上述方法。

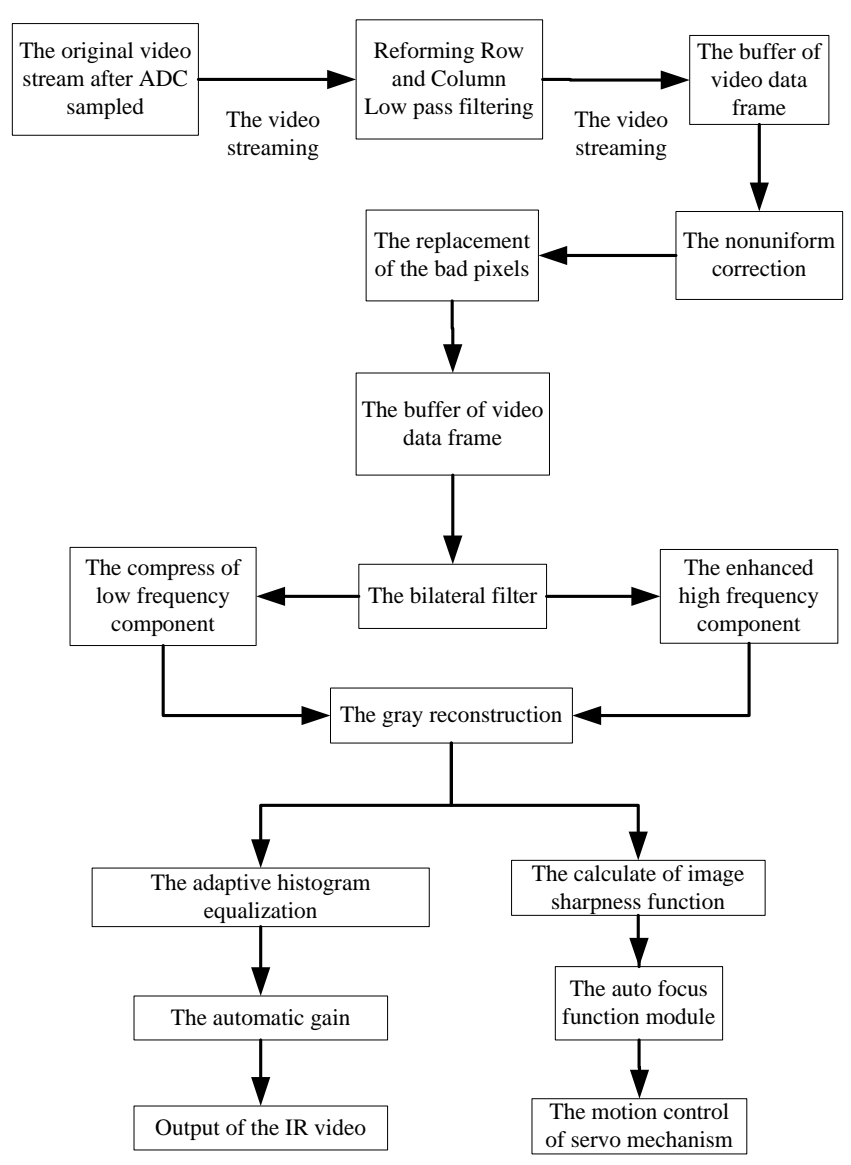

图 3 红外成像视频的数据流图

Fig.3 Data flow chart of the infrared imaging video 


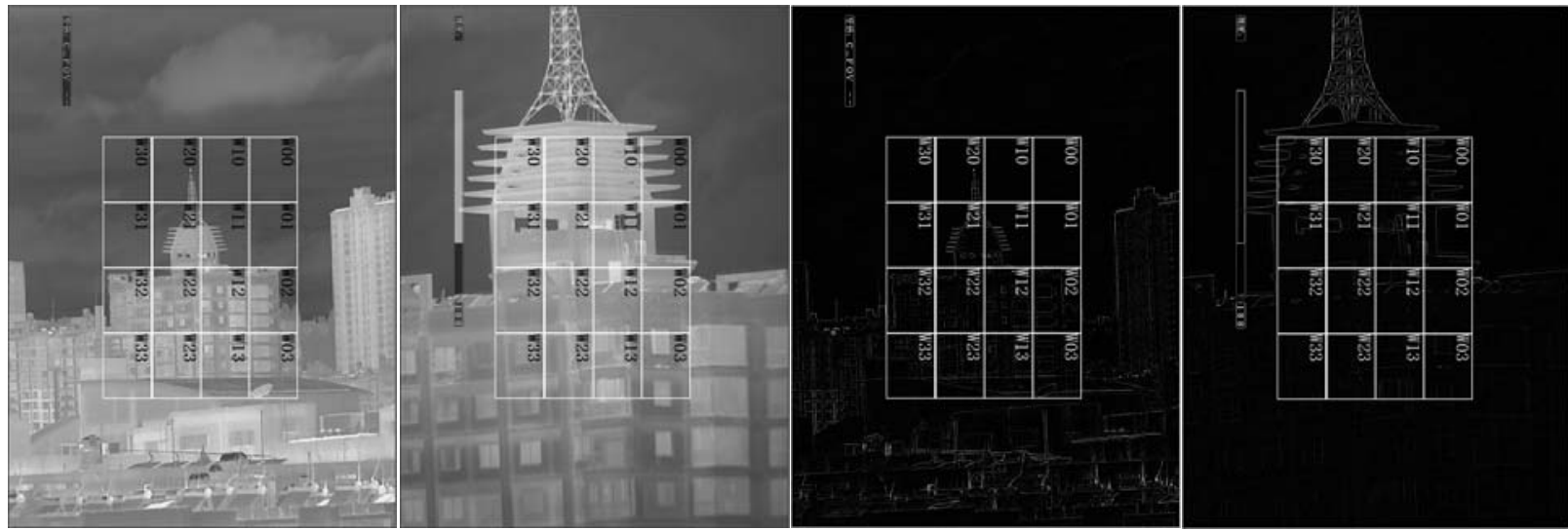

(a) 大视场下的热像图 (b) 小视场下的热像图 (c) 大视场下的拉普拉斯算子滤波后图像 (d) 小视场下的拉普拉斯算子滤波后图像

(a) The thermal image in wide field (b) The thermal image in narrow field (c) The image filtered by Laplace operator in wide field (d)The image filtered by Laplace operator in small field

\section{图 4 热像的图像清晰度评价函数中心窗口细分的示意图}

Fig.4 Schematic diagram of image definition evaluation function center window subdivision for a certain type of thermal image

\subsection{1 图像清晰度评价函数噪声的估计}

通过对图像清晰度评价函数噪声合理估计, 加 强了快速爬坡搜索算法反向或结束的约束条件，可 以提高算法的精度和可靠性。其理论方法简述如 下：对于一个前期经过滤波的、稳定的数字成像系 统, 我们可以将其近似地看做, 一个以目标场景为 输入, 图像清晰度评价函数为输出的一个惯性系 统, 目标场景输入如果是一个平稳随机过程, 其输 出也应该是一个平稳随机过程, 显然大多数连续, 慢速变化场景的成像系统满足上述条件。由于系统 存在微小的震动、背景噪声或计算误差, 图像清晰 度评价函数采样值表示为 $D^{\prime}=D+N_{D}$, 其中 $N_{D}$ 定义 图像清晰度评价函数局部起伏波动的噪声, 区别于 数字图像的本征噪声, $D$ 表示一个局部平滑的图像清 晰度评价函数。

一个实际的图像清晰度评价函数曲线可由两者 的叠加来表示。在自动对焦前或后的过程中, 图像 清晰度评价函数值通常是一个随视频同步信号同步 采样计算值系列 $D^{\prime}[N]$, 显然, 我们可以根据采样序 列 $D^{\prime}[N]$ 的均值或高阶项来估计局部噪声 $N_{D}$ （局部峰 值) 的大小。例如, 在噪声高斯分布情况下, 通过 $D^{\prime}[N]$ 的序列计算, 我们可以得到 $N_{D}$ 均值 $\mu$ 和方差 $\sigma$, 例如, 将噪声绝对值的最大值区间估计为 $\{\mu-3 \sigma, \mu$ $+3 \sigma\}$ （不同的系统, 可更具不同的应用需求或置信 度要求, 合理调整估计区间), 将得到一个大约 99.74\%的置信空间（其中，置信度 $P=\left\{\left|N_{D}-\mu\right| \leqslant 3 \sigma\right\}$ $\approx 0.9974$ )。

若将爬坡算法由 $D_{N^{\prime}-1}<D_{N}{ }^{\prime}>D_{N^{\prime}+1}$, 再加强一个 条件: $\Delta D^{\prime}=\mu+3 \sigma, \quad$ （其中 $\Delta D^{\prime}=\left|D_{N}{ }^{\prime}-D_{N-1}{ }^{\prime}\right|$ ），满 足此条件下爬坡过程有效, 则爬坡的过程, 就可以
跳出图像清晰度评价函数局部噪声峰峰值的区间（噪 声阈值)完成算法，并有 99.74\%置信度保证其结果的 有效性。

在本文的具体设计中, 将涉及实现自动对焦功 能实现的软件功能模块, 部署于成像系统中进行图 像处理的 FPGA 内部, 其中嵌入了一个 32 位 MCU 软 核处理器。上述软硬件架构, 有较强的计算和图像 处理能力, 更有利于保证自动对焦功能实时性能的实 现。考虑到自动对焦技术对于红外场景适应的多样 性, 实际应用要求 $N_{D}$ 能自适应地调整和估计, 本文 构建了一个输入 $D^{\prime}[N]$ 、最小均方误差 $N_{D}$ 为设计约束 的自适应数字滤波器, 可实现对 $N_{D}$ 的估计。在自动 调焦前, 固定的场景属于平稳随机过程, 滤波器类 似于维纳滤波器结构的数字滤波器, 以 $N_{D}$ 最小均方 误差准则构建数字滤波器, 估计量记为 $N_{D}{ }^{\prime}$, 并以当 前数字滤波器参数作为滤波器的初始值。当调焦机 构在自动调焦功能工作模式下, 输入变化为非平稳随 机过程, 滤波器进化为一个类似于卡尔曼滤波器结构 的自适应数字滤波器, 并实时调整滤波器序数矩阵的 参数值, 而得以实现上述自适应功能。采用上述的设 计优化方法, 额外带来的一个好处是, 确定了算法的 有效性的置信度后，也就确定图像清晰度评价函数局 部噪声阈值区间。若定义调焦步长为 $\Delta F$, 最小步长 (表 示为 $\operatorname{MIN}(\Delta F)$, 其中, $\left.\Delta F=F_{N}-F_{N^{-1}}\right)$ 也可以确定, 即自动调焦最小步长选取应大于等于, 引起图像清晰 度评价函数最小的改变量: $\mu+3 \sigma$ 。

为了验证上述设计的正确性, 分别在实施策略 前后, 各取 1000 次实验样本空间来验证设计的准确 性和可行性，在 1000 次红外成像系统自动对焦实验 中详细记录每次对焦清晰的次数。在红外成像系统 
未引入策略实施前, 在 1000 次实验样本中, 其中有 916 次执行完成自动对焦后, 并达到清晰位置 (命 中), 另外 84 次完成对焦后不能达到清晰位置（未 命中），成功命中率为 $91.6 \%$; 在成功引入策略实施 后, 在 1000 次有实验样本中有 982 次执行完成自动 对焦后达到清晰位置, 只有 18 次完成对焦后不能达 到清晰位置, 成功命中率为 $98.2 \%$ 。由此可见, 在实 施本文设计策略后, 自动对焦的成功命中率提高了 6.6 个百分点, 实验数据结果证明了本文针对红外成 像系统所采用的策略设计准确、可靠。该策略的实 施, 将有效地抑制图像清晰度评价函数的噪声, 并 大幅提升自动对焦功能的成功命中率。

\subsection{2 爬坡算法中反馈量的引入}

若将 $S_{N}=\left(D_{N}{ }^{\prime}-D_{N-1}{ }^{\prime}\right) /\left(D_{N}{ }^{\prime} \times\left(F_{N}-F_{N-1}\right)\right)$, 作为图 像清晰度评价函数归一化后, 相对焦距的变化量近似 值引入, 定义为灵敏度 (图像清晰度评价函数随调焦 变化量的敏感度), 并以此作为爬坡算法的反馈量, 将 有利于爬坡算法的收敛。 $S_{N}$ 及其高阶项也可用于估计 爬坡搜索算法的步进步长, 有利于算法的快速收玫。 例如, 在本文具体设计中, 当 $\Delta D>5(\mu+3 \sigma)$ (即 $\Delta D$ 远 大于 $(\mu+3 \sigma))$ 时, 可取 $\Delta F>\left((\Delta D-\mu-3 \sigma) \times D_{N}{ }^{\prime} / S_{N}\right)$ 对 $\Delta F$ 进行估计, 当 $\Delta D$ 不满足上述条件时, 最小调焦 步长的估计为; $\Delta F_{\mathrm{MIN}}=\left((\mu+3 \sigma) \times D_{N}{ }^{\prime} / S_{N}\right)$, 实际的最 小调焦步长应大于此估计值。

以实验验证上述的优化方法, 分别在实施策略 前后, 取 1000 次实验样本空间, 以平均迭代次数考 察算法的收玫速度。实施前, 爬坡算法平均迭代次 数为 7 ; 实施后, 爬坡算法平均迭代次数为 5 。实验 表明，上述设计方法有利于爬坡算法的收敛。

\subsection{3 分段标定并压缩对焦搜索的范围}

本文涉及的红外成像系统应用于军用背景, 要 求有较宽的工作温度范围, 较大视场变倍比, 于是 要求与之匹配的光学调焦系统也具有相应较长的调 焦行程。同时考虑到红外成像光学系统的齐焦性随 温度漂移较大, 其温度漂移特性大多稳定的特点, 如果在自动对焦前, 通过实验方法, 根据系统应用 要求的实际景深, 可分段标定其在各种温度的焦 深, 就能有效压缩自动对焦搜索的有效行程, 以提 高自动对焦功能的实时性能。通过实验来验证分区 的有效性, 具体实施方法如下, 结合红外非均匀校正 中两点校正的参数标定方法, 将设备的全温工作范围 $\left(-50^{\circ} \mathrm{C} \sim+70^{\circ} \mathrm{C}\right)$ 化分为 7 个温区, 压缩后的自动对 焦搜索范围与全温范围的对比示意如图 5 所示, 试验 数据如表 2 所示。在本实验方案中, 调焦系统全温调 焦行程总长为 $15 \mathrm{~mm}$, 最小调焦步进 $20 \mu \mathrm{m}$ 。未分段
前, 系统每次自动对焦都将在 $0 \sim 15 \mathrm{~mm}$ 的调焦行程 中遍历一遍, 从而确定最佳调焦点; 压缩对焦后最 长的自动对焦搜索范围 (比如温区 6) 比全温搜索范 围大概压缩了 $60 \%$ 的长度, 效率就提高了 $60 \%$ 以 上, 在实际工作中, 系统每次对焦之前会读取系统 当前的环境温度, 根据不同的温度, 在自动对焦时 选择不同的温区进行对焦。在本次试验过程中, 我 们同样取 1000 次有效实验样本来进行实际测试。

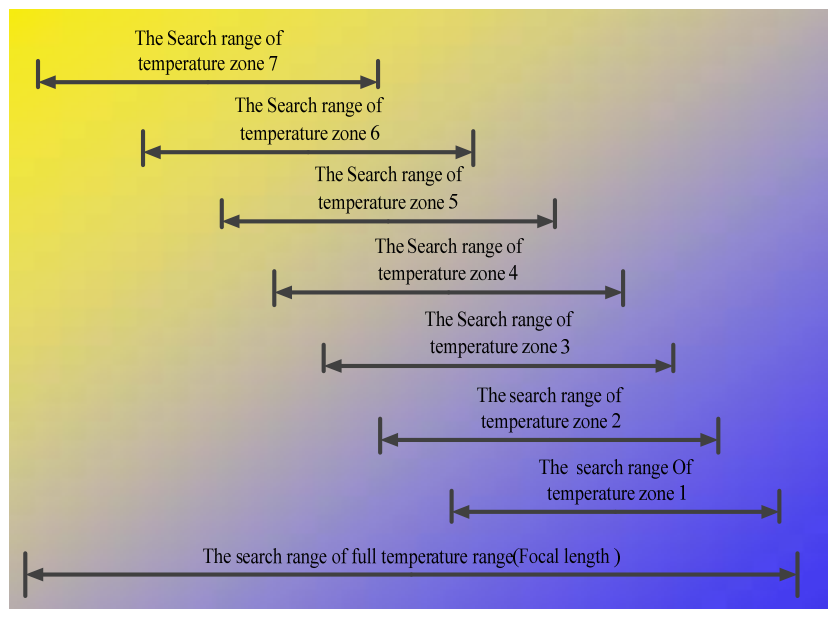

图 5 温区分段压缩后的搜索范围与全温的搜索范围对比示意图

Fig.5 The comparison chart of the search range of temperature region after segmented compression with that of full temperature

通过自动对焦系统实施压缩对焦策略前后的实 验数据分析, 当系统采用了压缩对焦策略之后, 系 统在全温度范围内自动对焦的有效时长从 $2.6 \mathrm{~s}$ 提高 到 $1.2 \mathrm{~s}$, 自动对焦的速度提高了 1.16 倍。

分段标定压缩的自动对焦搜索范围的方法, 能大 幅地压缩自动对焦搜索范围, 以提高其功能实时性。 但其适用的条件也是有一定约束限制的。其一, 该设 计策略主要适用于更宽的温度工作范围, 存在温度 有较大漂移的成像系统; 其二, 其方法有效性和效率 与成像系统的应用要求密切相关, 例如本文设计的红 外成像系统运用于无人机的观瞄和导航, 其飞行高度 很大程度将决定了有效的景深范围, 自然分段压缩后 的自动调焦搜索范围将有大幅的压缩; 其三, 其方法 策略是针对于红外成像系统的齐焦性随温度漂移较 大的特点而提出的, 其更适用于多视场、大变倍 比、长焦距调焦行程的红外成像系统。

\section{2 结论}

本文在某型号无人机机载红外热像仪的工程化 应用中, 采用了上述优化方法进行了自动对焦功能设 计, 其应用效果验证了优化方法的有效性。本方法除 


\section{表 2 实施策略前后自动调焦平均用时对比表}

Table 2 Comparison of average auto focusing time before and after implementation of the strategy

\begin{tabular}{|c|c|c|c|c|c|c|c|c|c|c|}
\hline \multirow{2}{*}{ The AF finished time/s } & \multicolumn{10}{|c|}{ The calibration temperature $/{ }^{\circ} \mathrm{C}$} \\
\hline & $-50^{\circ} \mathrm{C}$ & $-35^{\circ} \mathrm{C}$ & $-20^{\circ} \mathrm{C}$ & $-5^{\circ} \mathrm{C}$ & $+10^{\circ} \mathrm{C}$ & $+25^{\circ} \mathrm{C}$ & $+40^{\circ} \mathrm{C}$ & $+55^{\circ} \mathrm{C}$ & $+70^{\circ} \mathrm{C}$ & $-50^{\circ} \mathrm{C} \sim+70^{\circ} \mathrm{C}$ \\
\hline $\begin{array}{l}\text { The average time of } \\
\text { autofocus completion (no } \\
\text { temperature calibration } \\
\text { strategy applied) }\end{array}$ & 2.70 & 2.62 & 2.53 & 2.61 & 2.68 & 2.51 & 2.55 & 2.64 & 2.59 & 2.60 \\
\hline $\begin{array}{l}\text { The average time of } \\
\text { focusing completion } \\
\text { (applying temperature } \\
\text { calibration strategy) }\end{array}$ & 1.28 & 1.21 & 1.15 & 1.30 & 1.27 & 1.12 & 1.24 & 1.11 & 1.13 & 1.20 \\
\hline
\end{tabular}

了能有效提高图像清晰度评价函数的信噪比、灵敏 度、有效性，还能通过温区标定的方法压缩自动对焦 搜索范围，优化爬坡搜索算法的收敛速度，提升自动 对焦实时性能。然而, 本文对自动对焦技术在红外成 像系统中设计的优化方法，更多地局限于软件算法的 思想。实际应用中, 作为红外成像的光机要素, 诸如 红外探测器的性能, 调焦伺服机构运动速度、精度, 及光学设计的集成度等方面, 仍然是制约自动对焦的 技术瓶颈。期待随其相关硬件工艺及其综合制造能力 的提升, 红外成像系统中的自动对焦技术, 将取得更 为普遍而高效的应用。

\section{参考文献:}

[1] 何炳阳, 张智诠, 杨秋实, 等. 热成像系统自适应自动对焦算法 [J]. 激光与红外, 2017, 47(10): 1199-1263.

HE Bingyang, ZHANG Zhiquan, YANG Qiushi, et al. Adaptive auto-focusing algorithm of thermal imaging system[J]. Laser \& Infrared, 2017, 47(10): 1199-1263.

[2] 林忠, 黄陈蓉, 卢阿丽. 自动对焦综合性能指标及一种基于双向两次 下降的自动对焦算法[J]. 科学技术与工程, 2015, 15(32): 175-180.

LIN Zhong, HUANG Chenrong, LU Ali. Auto-focus compressive performance evaluation and an auto-focus algorithm based on two-way twice descent[J]. Science Technology and Engineering, 2015, 15(32): 175-180.

[3] 郝争辉, 张学松, 王高, 等. 基于边缘轮廓线提取的自动对焦评价函 数 $[\mathrm{J}]$. 红外技术, 2018, 40(2): 170-175.

HAO Zhenghui, ZHANG Xuesong, WANG Gao, et al. Auto focusing evaluation function based on edge contour extraction[J]. Infrared Technology, 2018, 40(2): 170-175.

[4] 王烨茹, 冯华君, 徐之海, 等. 一种覆盖范围可调的变频梯度自动对 焦评价函数 [J]. 红外与激发工程, 2016, 45(10): 258-263.

WANG Yeru, FENG Huajun, XU Zhihai, et al. An adjustable coverage range autofocus evaluation function using gradient operator with variable
frequency[J]. Infrared and Laser Engineering, 2016, 45(10): 258-263.

[5] 尤玉虎, 刘通, 刘佳文. 基于图像处理的自动对焦技术综述 [J]. 激光 与红外, 2013, 43(2): 132-136.

YOU Yuhu, LIU Tong, LIU Jiawen. Survey of the auto-focus methods based on image processing[J]. Laser \&Infrared, 2013, 43(2): 132-136.

[6] 李少华. 红外成像系统自动调焦功能的设计与实现[D]. 武汉: 华中科 技大学, 2013.

LI Shaohua. Design \& Realization of Auto-focusing in FPGA-based Infrared Imaging System[D]. Wuhan: Huazhong University of Science \& Technology, 2013.

[7] 王红伟, 王加庆, 吴南健. 基于高速视觉目标跟踪系统的自动调焦算 法的设计 [J]. 光电系统, 2018, 39(5): 716-721.

WANG Hongwei, WANG Jiaqing, WU Nanjian. Design of automatix focusing algorithm based on high speed vision object tracking system[J]. Semiconductor Optoelectronic, 2018, 39(5): 716-721.

[8] 马宣. 能够自动对焦的红外成像系统软件的设计 [D]. 成都: 电子科技 大学, 2014.

MA Xuan. The Design of an Auto-Focusing Infrared Imaging System[D]. Chengdu: University of Electronic Science and Technology of China 2014.

[9] 王建瑞, 邓华秋, 陈参宁. 基于红外图像的自动调焦技术 $[\mathrm{J}]$. 光电技 术应用, 2018, 33(3): 1-5.

WANG Jianrui. Auto focusing technology based on infrared image[J]. Electro-Optic Technology Application, 2018, 33(3): 1-5.

[10] 王剑华, 邓华秋, 陈参宁. 数字自动对焦中的搜索算法研究[J]. 传感 器与微系统, 2012, 31(5): 51-54.

WANG Jianhua, DENG Huaqiu, CHEN Canning. Research on search algorithm for digital auto-focus[J]. Transducer and Microsystem Technologies, 2012, 31(5): 51-54.

[11] HE J, ZHOU R, HONG Z. Modified fast climbing search auto-focus algorithm with adaptive step size searching technique for digital camera[J]. IEEE Transactions on Consumer Electronics, 2003, 49(2): 257-262. 